\title{
ISO observations of symbiotic stars
}

\section{HM Sge}

\author{
H. Schild ${ }^{1}$, S. P. S. Eyres ${ }^{2,3, \star}$, A. Salama ${ }^{4}$, and A. Evans ${ }^{2}$ \\ 1 Institut für Astronomie, ETH-Zentrum, 8092 Zürich, Switzerland \\ 2 Department of Physics, Keele University, Keele, Staffs, ST5 5BG, UK \\ 3 Astrophysics Research Institute, Liverpool John Moores University, Twelve Quays House, Egerton Wharf, \\ Birkenhead, CH41 1LD, UK \\ 4 ISO Data Centre, ESA Astrophysics Division, Villafranca del Castillo, Spain
}

Received 2 October 2000 / Accepted 14 August 2001

\begin{abstract}
We present ISO SWS and LWS observations of the symbiotic star HM Sge. The infrared emission longward of $\sim 5 \mu \mathrm{m}$ is dominated by a silicate dust shell. There is a number of high ionization emission lines superimposed on the dust continuum. The ISO continuum spectra can be fitted with a single, optically thick silicate dust shell but such a model does not fit other known properties of this system, such as the observed $J H K$ colours, the optical visibility of the red giant and the occurrence of a dust obscuration event. We favour a two component model in which in addition to a partial Mira dust shell, a second dust component is associated with the hot source. This second shell is located at the interface between the Mira dust shell and the dust free region carved out by the hot component. This picture corresponds closely to that deduced independently from radio observations. The two dust components are very different. The dust envelope associated with the Mira is very extended and optically thin, whereas the second dust component is geometrically rather thin but dust rich. The model not only fits the ISO data reasonably well, but is also compatible with other IR properties of HM Sge. The thin dust shell contributes strongly to the dust emission over most of the ISO range. It may be the combination of the two shells that makes a symbiotic a D type. The general agreement of the present models with ISO observations indicate that radiation (rather than collisions) as the main dust heating mechanism is sufficient.
\end{abstract}

Key words. binaries: symbiotic - circumstellar matter - stars: individual: HM Sge - stars: winds, outflows infrared: stars

\section{Introduction}

\subsection{Preamble}

Symbiotic stars are a heterogeneous group of objects, having in common the presence of a hot $\left(\sim 10^{5} \mathrm{~K}\right)$ and a cool (e.g. a red giant or Mira) component (see Mikołajewska 1997, for a review). They have been postulated as possible proto-planetary nebulae (e.g. Lutz et al. 1989; Corradi 1995) and as precursors to Type Ia supernovae (Munari \& Renzini 1992). They are also closely related to classical and recurrent novae and indeed, a small subset of symbiotics are known to have undergone a nova-like eruption. The cause of the eruption is most likely the same as that for classical novae, namely thermonuclear runaway on the surface of the hot component.

\footnotetext{
Send offprint requests to: A. Evans,

e-mail: ae@astro.keele.ac.uk

* Present address: Centre for Astrophysics, University of Central Lancashire, Preston, PR1 2HE, UK.
}

Symbiotics are further subdivided on the basis of their infrared (IR) properties, into S-types (in which the stellar emission dominates in the IR) or D-types (in which dust emission dominates). The cool component in symbiotics may be oxygen- or carbon-rich, and we would expect the dust (in D-types) to be correlated with abundances in the underlying star.

A number of symbiotic stars were observed by the Infrared Space Observatory ISO (see Eyres et al. 1998; Schild \& Vogel 1998; Schild et al. 1999, for some preliminary discussion) and we describe here observations of HM Sge with ISO (Kessler et al. 1996).

\subsection{HM Sge}

HM Sge is a D-type symbiotic, in which the cool component is a M 7 Mira (Mürset \& Schmid 1999) with 527 day pulsation period (Munari \& Whitelock 1989). It is one of the best-studied of the symbiotics and, in common with a number of other symbiotic systems (e.g. RR Tel, 
V1016 Cyg), HM Sge has undergone a nova-like eruption (in 1975).

The distance of HM Sge has been the subject of much discussion. Whitelock (1987) suggests a distance $D=2.3 \mathrm{kpc}$ by applying the Period-Luminosity relation for LMC Miras to the Mira component in the HM Sge system (see e.g. Feast et al. 1989; and Feast 1996 for a comparison between LMC and Galactic $P-L$ relationships for Miras). Mürset et al. (1991) suggest a distance of $2.9 \mathrm{kpc}$, with reddening $E(B-V)=0.65$; a smaller distance $(1.3 \mathrm{kpc})$ and $E(B-V)=0.35$ is deduced by Eyres et al. (2001, see below).

Near IR photometry is presented by Munari \& Whitelock (1989) and by Munari et al. (1992). Munari \& Whitelock find that, superimposed on the normal pulsations of the Mira, the visual and JHK light curves showed distinct fading and reddening, starting around 1985, which they interpreted as a dust-obscuration event. Intermediate resolution $K$-band spectroscopy taken in 1990 showed a flat continuum and in particular, no trace of stellar $\mathrm{CO}$ absorption features (Schild et al. 1992).

IRAS observations of HM Sge are discussed by (amongst others) Anandarao et al. (1988), who model the IRAS LRS $(8-23 \mu \mathrm{m})$ spectrum in terms of an isothermal $\left(T_{\text {dust }} \simeq 380 \mathrm{~K}\right)$ silicate dust shell which obscures the Mira; they further find that a second, cooler $\left(T_{\text {dust }} \simeq\right.$ $70 \mathrm{~K})$ shell is needed to fit the far-IR $(\lambda \geq 25 \mu \mathrm{m})$ IRAS photometry. They further conclude that, in the case of symbiotics displaying nova-like behaviour, the dust-shell temperatures are anti-correlated with the time since the last eruption.

Spectropolarimetry of HM Sge is described by Schmid et al. (2000). They find that significant changes in the spectrum have occurred between 1995 and 1998. In the near IR the spectrum of the Mira has appeared, but the nebular emission line spectrum is essentially unchanged. They concluded that these changes indicate that the dust content in the environment of HM Sge is rapidly dimininshing, and that the dust obscuration period which began in 1985 has come to an end. They further find that the Mira is intrinsically polarized at the $0.5 \%$ level, and that there is polarization structure across the $\mathrm{TiO}$ bands. They also determimed the orientation of the binary axis, and found it to be parallel or perpendicular to certain features of the surrounding nebulosity.

HM Sge has been extensively studied at radio wavelengths. Kwok et al. (1984) interpreted their radio data in terms of the interaction between the winds of the hot and cool components (see in particular their Fig. 5); they further concluded that the eruption in 1975 was merely the clearing of the circumstellar environment and the subsequent ionization of the cicumstellar shell.

More recent radio observations are discussed by Richards et al. (1999), who analysed VLA and MERLIN images. They also found the data to be consistent with a colliding winds model, in which the cool outflow from the giant component interacts with the hot, fast wind from the hot component. This predicted a projected binary
Table 1. Log of ISO observations.

\begin{tabular}{lcccc}
\hline & $\begin{array}{c}\lambda \text { range } \\
(\mu \mathrm{m})\end{array}$ & Date & $\begin{array}{c}\text { UT start } \\
\text { time }\end{array}$ & $\begin{array}{c}\text { On-target } \\
\text { time }(\mathrm{s})\end{array}$ \\
\hline SWS & $2.4-45.2$ & $1996-10-01$ & $02: 02: 39$ & 1140 \\
LWS & $43.1-195.7$ & $1996-10-01$ & $02: 22: 23$ & 822 \\
LWS $^{*}$ & $43.1-195.7$ & $1996-10-01$ & $02: 37: 01$ & 823 \\
SWS & $2.4-45.2$ & $1997-05-16$ & $01: 37: 43$ & 1912 \\
\hline
\end{tabular}

* Background observation.

separation of $25 \mathrm{AU}$; for reasonable stellar masses, this implies an orbital period $\gtrsim 100$ years. The radio images showed the central regions of the nebula filling, suggesting ongoing but irregular mass loss from the central binary. Extended non-thermal radio emission was also confirmed, and shown to be consistent with synchrotron emission in a $\mu \mathrm{T}$ magnetic field.

HST imaging of the nebula associated with HM Sge is described by Eyres et al. (2001). These authors determine the variation in extinction across the nebula, and hence the distribution of circumstellar dust, over a region $\sim 1 \operatorname{arcsec}^{2}$. They find that the extinction seems to be clumpy, and argue that the greatest concentration of dust is associated with the cool component wind, while a deficiency of dust is associated with the central binary and wind interaction region. The maximum $E(B-V) \simeq 1$, corresponding to an optical depth in the visual of $\tau_{\mathrm{v}} \simeq 3$ for a reasonable reddening law; since this estimate is based on [O III] line ratios it must include both circumstellar and foreground extinction. Furthermore, they deduce a projected binary angular separation of $40 \pm 9$ mas, consistent with the results of Richards et al. (1999) if the distance is $\sim 600 \mathrm{pc}$.

The X-ray emission from HM Sge is discussed by Mürset et al. (1997) who find X-ray emission arising in shocked gas at $T_{\text {gas }} \simeq 10^{6.6} \mathrm{~K}$; the plasma luminosity is $10^{-0.36} L_{\odot}$. The hot (stellar) component is found to have effective temperature $T_{*} \simeq 200000 \mathrm{~K}$ and $L_{*} \simeq 9200 L_{\odot}$; the corresponding radius is $\simeq 0.08 R_{\odot}$, characteristic of a white dwarf.

\section{Observations}

The observations described here were made with the ISO Short Wavelength Spectrometer (SWS; de Graauw et al. 1996; Salama et al. 1997) and Long Wavelength Spectrometer (LWS; Clegg et al. 1996; Burgdorf et al. 1997). The two observations were obtained on ISO revolutions 319 (1996 October 1 = JD 2450357.6) and 547 (1997 May $16=$ JD 2450584.6). The visual light curve for HM Sge at this time, obtained from the VSNET archive (VSNET 1999), is shown in Fig. 1. Our observations were 228 days apart, while the period of the Mira variable in the HM Sge system is 527 days, corresponding to a phase shift of 0.43. Using the ephemeris of Yudin et al. (1994) we find that the observation on ISO revolution 319 was taken at phase 0.65 (i.e. shortly after the photometric maximum 


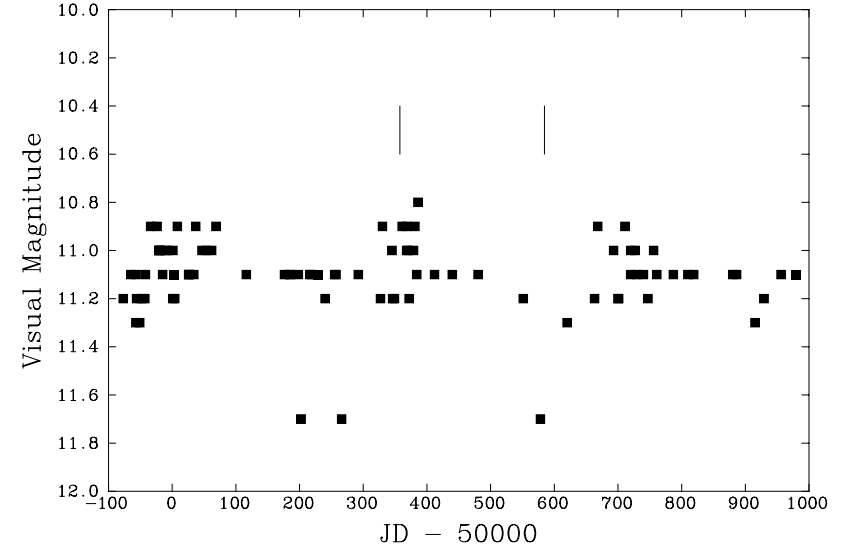

Fig. 1. Visual light curve of HM Sge at the time of the ISO observations; data from VSNET (1999). Vertical lines indicate times of the ISO observations presented here.

in the Mira variations) and that on revolution 547 was at phase 1.08, close to Mira minimum.

The observation $\log$ is given in Table 1 . The observations employed a full grating scan with the SWS using the Astronomical Observational Templates (AOT) SWS-01 Speed 1 and LWS-01; a background LWS observation was carried out at a position $6^{\prime} \mathrm{N}-\mathrm{W}$ of the position of the target. Background emission is detectable above $60 \mu \mathrm{m}$; it rises in intensity from shorter wavelengths and reaches a maximum $\left(\sim 4 \times 10^{-15} \mathrm{~W} \mathrm{~m}^{-2} \mu \mathrm{m}^{-1}\right)$ at $90 \mu \mathrm{m}$, then slowly declines, reaching a constant value of $\sim 2 \times$ $10^{-15} \mathrm{~W} \mathrm{~m}^{-2} \mu \mathrm{m}^{-1}$ above $130 \mu \mathrm{m}$. The background is stronger than the emission from HM Sge above $80 \mu \mathrm{m}$, and dominates completely above about $100 \mu \mathrm{m}$. HM Sge is detectable up to $110 \mu \mathrm{m}$. The [C II] $\lambda 158$ line is clearly present, but is entirely of interstellar origin.

The data are shown in Fig. 2. The dust emission longward of $\sim 2 \mu \mathrm{m}$ is clearly visible, and the silicate features at $9.7 \mu \mathrm{m}$ and $18 \mu \mathrm{m}$ are also prominent. There may be differences in the spectrum around the $18 \mu \mathrm{m}$ silicate feature at JD 357.6 and JD 584.6 but they are marginal. If the changes are real, it would indicate that the optical depth of the dust shell decreased, which would be consistent with recent optical observations (Schmid et al. 2000) in which the $\mathrm{TiO}$ bands in the Mira are seen for the first time. The implication is that the Mira component is slowly becoming visible in the optical. This effect most likely arises from the thinning out of the dust shell, as the orbital period is too long for it to be an orbital effect. However, while there is no conclusive evidence that the dust emission has changed, there are almost certainly changes in the near IR $(\lesssim 2 \mu \mathrm{m})$ which are likely due to Mira variability.

There are also several emission lines superimposed on the dust continuum. There is no evidence that the emission line strengths changed between the two ISO observations. This would be consistent with the view that any changes in the dust emission arise from changes in the Mira component, while the hot component - which powers the emission lines - is constant.

\section{The dust emission}

The ISO SWS spectra of HM Sge are clearly dominated by the emission of a dust shell. The LWS spectrum also shows the decreasing emission of the dust up to $60 \mu \mathrm{m}$ but beyond that wavelength, background radiation becomes important (see Sect. 2); accordingly we confine the modelling to wavelengths $<50 \mu \mathrm{m}$.

We first note the absence of any conspicuous molecular absorption features below $5 \mu \mathrm{m}$ (e.g. the CO fundamental at $\sim 4.8 \mu \mathrm{m}$ ) that one might expect to see in the near IR spectrum of a Mira variable. This, together with the fact that HM Sge is a D-type symbiotic, suggests that there must be appreciable dust emission down to the shortest wavelengths observed $(\lesssim 3 \mu \mathrm{m})$.

\subsection{Dust models}

In a first attempt to interpret these spectra we have used the DUSTY code (Ivezić \& Elitzur 1997), in which the radiation from a central source of radiation is transported outwards through a spherically symmetric dusty envelope. Processes that are included are thermal emission, scattering and absorption. We acknowledge that DUSTY calculates the emission of spherically symmetric, complete dust shells and that the geometries we consider are for the present constrained by the program; clearly more realistic geometries should in due course be explored but are beyond the scope of the present work.

Given the nature of the Mira in HM Sge (see Sect. 1.2) we chose an input model atmosphere with $T_{\text {eff }}=3000 \mathrm{~K}$ and $\log g=-0.29$. The Mira temperature was kept constant and the stellar spectrum was taken from the library of stellar spectra by Lejeune et al. (1997). In the case of $\mathrm{M}$ giants, these are based on model atmospheres of Fluks et al. (1994).

The density law in the dust shell $\left[n(r) \propto r^{-x}\right.$, where $n$ is the number density of grains] is a free parameter and we explored models with $1.0<x<2.0$.

In all models the dust particles have a standard size distribution as described by Mathis et al. (1977), $n(a) \propto$ $a^{-3.5}$ with minimum and maximum grain sizes of $0.005 \mu \mathrm{m}$ and $0.25 \mu \mathrm{m}$ respectively. We use silicate dust with optical properties given by Ossenkopf et al. (1992).

\subsubsection{Single shell}

We first tried to fit a single shell model to our ISO data and it is indeed possible to find a model that is in reasonable agreement with both observations (Fig. 2). The corresponding dust shell parameters are listed in Table 2. The density slope $x$ of 1.8 is close to what is expected from a freely expanding extended atmosphere and the dust condensation temperature $T_{\text {cond }}$ of $1600 \mathrm{~K}$ is slightly higher than that which is typical for this type of object (e.g. Lobel et al. 2000). The dust shell is large with an outer radius $10^{3}$ times the inner radius and also the optical thickness at $5500 \AA, \tau_{\mathrm{v}} \approx 29$ is very large. In fact this 


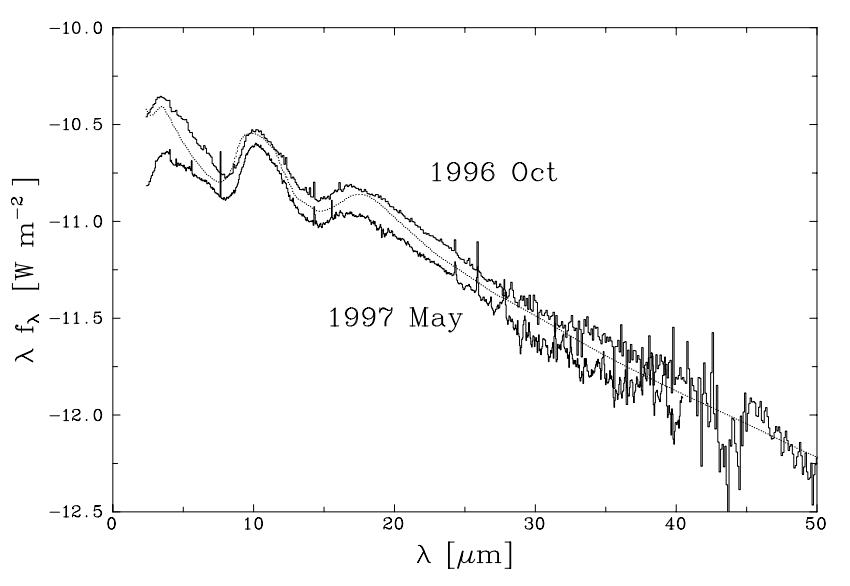

Fig. 2. ISO observations (thick lines) with single shell dust model (thin line); see Sect. 3.1.1.

high optical depth poses a problem because the associated high extinction ( $\gtrsim 30 \mathrm{mag}$ ) for the Mira is incompatible with contemporaneous JHK photometry by Kamath \& Ashok (1999). In addition, as mentioned above, optical spectra from June 1998 show the TiO features of the red giant (Schmid et al. 2000). The true absorption column in the line of sight towards the Mira must therefore have been very much smaller. Although the dust distribution is probably clumpy (Eyres et al. 2001) it is unlikely that such large gradients can be maintained in a circumstellar medium that, in all likelihood, is highly turbulent. Alternatively, the radiation from the hot component must have evaporated or otherwise removed large parts of the Mira dust envelope such that the line of sight towards the Mira is now cleared of dust. Indeed it is very likely that the hot stellar component has removed large parts of the Mira dust envelope since its outburst. On the other hand, it was not the case that the optical thickness towards the red giant has steadily decreased - on the contrary a dust obscuration event started in 1985 (Munari \& Whitelock 1989) which lasted until recently.

In summary, although a single dust envelope model can be found that fits the ISO spectrum it is difficult to reconcile it with the above facts about the IR properties of HM Sge. It may also be too simplistic since the Mira has a hot companion star with its own wind which presumably leads to a highly compressed wind collision zone. We therefore also investigated a series of models with two dust components.

\subsubsection{Two shell models}

It is evident that the hot star will evacuate a cavity in the dusty wind of the red giant, either by ionisation or a wind by its own. At the interface between the giant wind and the cavity, there will be a heated layer of dust which has radial symmetry and which is centred on the hot component. We therefore explore a 2-component model in which each stellar component has its own dust shell; we further suppose that neither component heats the dust shell of the
Table 2. Summary of DUSTY fits to HM Sge data. Shell "M" is the dust shell associated with the Mira component, Shell "H" that associated with the hot component. $x$ is the exponent of the dust density law, $T_{\text {cond }}$ is the temperature at the inner edge of the dust shell, $Y$ is the ratio of outer-to-inner dust-shell radius, $\tau_{\mathrm{v}}$ is the dust shell optical depth in the visual; see text for details. Values marked with an asterix were pre-determined and fixed.

\begin{tabular}{l|c|cc}
\hline & Single shell & \multicolumn{2}{|c}{ Two shells } \\
\hline & & $\mathrm{M}$ & $\mathrm{H}$ \\
\hline Slope $x$ & 1.8 & 1.6 & $0^{*}$ \\
$T_{\text {cond }}(\mathrm{K})$ & 1600 & $1400^{*}$ & 800 \\
$Y$ & 1000 & 1000 & 1.1 \\
$\tau_{\mathrm{v}}$ & 29 & 1.5 & 13 \\
Inner radius $(\mathrm{cm})$ & $5.6 \times 10^{13}$ & $6 \times 10^{13}$ & $2.8 \times 10^{15}$ \\
Thickness $(\mathrm{cm})$ & $6 \times 10^{16}$ & $6 \times 10^{16}$ & $3 \times 10^{14}$ \\
Relative contribution & - & 10 & 1 \\
\hline
\end{tabular}

other. DUSTY calculates the emission of spherically symmetric, complete dust shells. In order to allow for partial shells we combine the two dust shells with a partition factor $p$ such that the observed flux

$F_{\text {obs }}=s\left(F_{\text {Shell H }}+p F_{\text {Shell M }}\right)$.

The scaling factor $s$ converts the model flux at the distance of HM Sge into the observed flux.

In order to limit the parameter space, we specify that the optical depth of the red giant dust shell should be small to take into account the fact that the Mira in HM Sge became optically observable shortly after our ISO observations. Its optical depth in the visual was therefore required to lie in the range $0.5<\tau_{\mathrm{v}}<3.5$. The dust condensation temperature was fixed at $1400 \mathrm{~K}$ because this represents a value typical for red giant dust shells (e.g. Habing 1996). We used a grid of 28 models for the dust shell associated with the Mira. The main free parameter was the slope of the density law.

We also imposed on the dust shell associated with the hot component the constraint that its thickness should not be excessively large $(Y=\{$ outer radius $\} /\{$ inner radius $\} \lesssim 5$ ) and therefore it could be approximated with a flat density distribution $(x=0)$. We explored dust condensation temperatures in the range $600 \mathrm{~K}$ to $2000 \mathrm{~K}$, and optical depth $\tau_{\mathrm{v}}$ in the range 1 to 50 . The hot component temperature was set to $200000 \mathrm{~K}$ (cf. Sect. 1.2). We calculated about 500 models for the shell associated with the hot component. The main free parameters were the shell thickness, optical depth and the dust condensation temperature.

For each combination the relative contributions of the two shells (partition factor $p$ ) was varied to match the slope of the observed spectrum. This factor describes the relative contributions (Mira dust shell/hot component dust shell) that gives the best agreement with observations. It reflects not only the relative size of the shells but also accounts for partial shells. For both shells the standard dust size distribution of Mathis et al. (1977) and the 


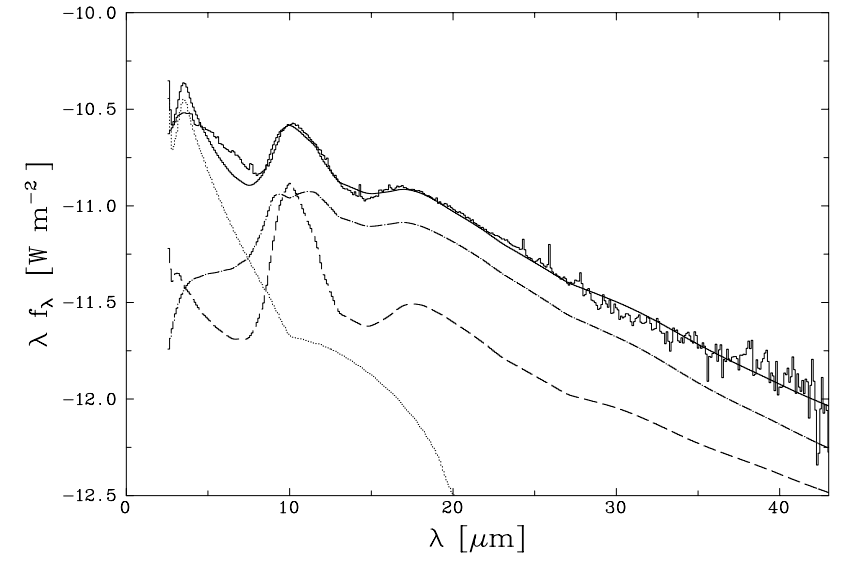

Fig. 3. Mean of ISO spectra of HM Sge with a model fit described in the text (see Sect. 3.1.2). Contributions to the model spectrum are: Shell $\mathrm{H}$ (associated with the hot component; dashed line), Shell M (associated with the Mira; dot-dashed). The dotted line is the red giant continuum.

Ossenkopf et al. (1992) optical properties of warm silicates were adopted.

In total some 420000 model combinations were generated and compared with the ISO spectra. The best model fit is obtained with the parametrisation given in Table 2, and the resulting model fit is shown in Fig. 3 together with the averaged SWS ISO spectra of HM Sge. The best fit is obtained with a partition factor $p$ of 10 and a scaling factor $s$ of $1.5 \times 10^{-11}$. The two resulting dust shells are very different: one is geometrically thick and optically thin and the other is geometrically thin and optically thick (see Table 2).

We note that this configuration, deduced from our ISO data, is consistent with that deduced by Kwok et al. (1984) for the interaction region between the winds of the hot and Mira components. In their model a silicate emission component is associated with the Mira wind and a geometrically thin 'interaction region' is roughly centred on the hot component. We can plausibly associate our optically thin, geometrically thick shell with the Mira wind, and the geometrically thin, optically thick shell with the interaction region. Their proposed geometry (see their Fig. 5) gives us confidence that our approach to the modelling of the ISO data, with two spherically symmetric dust shells, is valid.

We note that DUSTY uses scaling properties such that the stellar luminosities are not required as input parameters. In order to convert e.g. the inner radii onto an absolute scale these luminosities are needed. For the red giant we applied the period luminosity relation for Miras by Feast $(1996)$ and find $L_{\mathrm{cool}} \approx 5000 L_{\odot}$ and for the hot component we adopted the data of Mürset et al. (1997) which lead to $L_{\text {hot }} \approx 9200 L_{\odot}$. With these luminosities the respective absolute inner shell radii in Table 2 follow.

In Fig. 3 we also plot the flux contributions of the individual dust shells. Dust emission contributes over the full wavelength range, and starts to dominate above around $6 \mu \mathrm{m}$. Even at $3 \mu \mathrm{m}$ there is a contribution from the dust to the total emission, which is consistent with the apparent lack of molecular features from the Mira atmosphere at these wavelengths.

\subsection{Results}

As noted in Sect. 1.2, Eyres et al. (2001) deduce a maximum value $E(B-V) \simeq 1$ from the HST data. If we combine this with the foreground reddening of $E(B-$ $V) \simeq 0.35$ (Eyres et al. 2001), a circumstellar component $E(B-V) \simeq 0.65$ is implied. We see in Table 2 that our DUSTY modelling implies circumstellar extinction for Shell $\mathrm{M}$ of $\tau_{\mathrm{v}}=1.5$, implying a circumstellar $E(B-V) \simeq 0.5$ for a reasonable reddening law. Given the inherent uncertainties, this is gratifyingly close to the value in Eyres et al. (2001).

We also note that the large $\tau_{\mathrm{v}}$ for Shell $\mathrm{H}$ implies a high absorbing column, but such a thin shell is likely to be patchy and in fact provides a natural explanation for dust obscuration events.

The overall agreement between the DUSTY model and the observations is generally satisfactory. The greatest discrepancies occur at short wavelengths $(\lambda \lesssim 7 \mu \mathrm{m})$ and can certainly partly be attributed to the variability of the Mira. According to the ephemeris of Yudin et al. (1994) our observations were obtained at Mira phases 0.65 and 1.08. The Mira peaks at phase 0.4 and the minima occur (by definition) at phase 0.0. Our later ISO observation was thus obtained close to minimum phase (see Fig. 1) and our first ISO observation was taken shortly after photometric maximum. DUSTY generates model dust shells that are in equilibrium with the central star and improvements in the spectral fit may be possible if an averaged Mira spectrum were used as stellar input spectrum.

A second source of discrepancy may be related to our treatment of the dust shell associated with the hot component (Shell H). It is certainly much thinner than Shell M but it is also by no means clear whether its dust distribution is flat. Also the proximity of a wind collision zone with hard X-ray radiation may affect the dust properties and the optical constants of Ossenkopf et al. (1992) may not be entirely appropriate for the dust in this environment. For example, any newly-formed dust in the Mira wind may be processed in the hard radiation of the hot component. Indeed Eyres et al. (1998) have noted the similarity of the silicate feature in some symbiotic stars to those seen in some classical novae, where the radiation field is hard, and in which the $9.7 \mu \mathrm{m}$ silicate feature suggest some degree of crystallinity.

Further evidence for the effect of the hot component on the dust may be seen in the slope of the density distribution in the shell associated with the red giant. A steady state wind originating at the Mira and having a constant expansion velocity is expected to generate a density falling away with distance $r$ as $r^{-2}$. Our fit suggests a much flatter distribution, which might be a crude reflection of the hydrodynamical influence exercised by the hot companion. 
Table 3. Line fluxes in the SWS spectrum of HM Sge.

\begin{tabular}{ccccc}
\hline$\lambda(\mu \mathrm{m})$ & ID & Transition & \multicolumn{2}{c}{$\begin{array}{c}\text { Integrated Flux } \\
\left(10^{-15} \mathrm{~W} \mathrm{~m}^{-2}\right)\end{array}$} \\
\cline { 3 - 5 } & & & Rev 319 & Rev 547 \\
\hline 4.0522 & $\mathrm{H} \mathrm{I}$ & $5-4$ & $6.6 \pm 0.4$ & $5.8 \pm 0.6$ \\
4.33 & {$[\mathrm{Ni} \mathrm{v}]$} & & & \\
& {$[\mathrm{Cr} \mathrm{XVII}]$} & & $<7.7$ & $5.1 \pm 0.7$ \\
4.5295 & {$[\mathrm{Ar} \mathrm{VI}]$} & ${ }^{2} \mathrm{P}_{3 / 2}-{ }^{2} \mathrm{P}_{1 / 2}$ & $6.6 \pm 1.1$ & $8.7 \pm 1.8$ \\
5.6099 & {$[\mathrm{Mg} \mathrm{V}]$} & ${ }^{3} \mathrm{P}_{1}-{ }^{3} \mathrm{P}_{2}$ & $14.1 \pm 0.9$ & $16.4 \pm 1.6$ \\
6.4922 & {$[\mathrm{Si} \mathrm{VII}]$} & ${ }^{3} \mathrm{P}_{0}-{ }^{3} \mathrm{P}_{1}$ & $<5$ & $<5$ \\
7.6524 & {$[\mathrm{Ne} \mathrm{VI}]$} & ${ }^{2} \mathrm{P}_{3 / 2}-{ }^{2} \mathrm{P}_{1 / 2}$ & $74.0 \pm 0.6$ & $71.1 \pm 2.6$ \\
8.6106 & {$[\mathrm{Na} \mathrm{VI}]$} & ${ }^{3} \mathrm{P}_{2}-{ }^{3} \mathrm{P}_{1}$ & $7.2 \pm 0.6$ & $<3$ \\
9.5267 & {$[\mathrm{Fe} \mathrm{VII}]$} & ${ }^{3} \mathrm{~F}_{3}-{ }^{3} \mathrm{~F}_{2}$ & $3.7 \pm 0.5$ & $<3$ \\
10.5105 & {$[\mathrm{Si} \mathrm{IV}]$} & ${ }^{2} \mathrm{P}_{3 / 2}-{ }^{2} \mathrm{P}_{1 / 2}$ & $3.1 \pm 0.4$ & $3.0 \pm 0.3$ \\
12.8135 & {$[\mathrm{Ne} \mathrm{II}]$} & ${ }^{2} \mathrm{P}_{1 / 2}-{ }^{2} \mathrm{P}_{3 / 2}$ & $<5$ & $3.1 \pm 0.3$ \\
14.3217 & {$[\mathrm{Ne} \mathrm{V}]$} & ${ }^{3} \mathrm{P}_{2}-{ }^{3} \mathrm{P}_{1}$ & $9.7 \pm 0.7$ & $12.3 \pm 0.9$ \\
15.5551 & {$[\mathrm{Ne} \mathrm{III}]$} & ${ }^{3} \mathrm{P}_{1}-{ }^{3} \mathrm{P}_{2}$ & $6.1 \pm 0.8$ & $7.8 \pm 0.4$ \\
24.3175 & {$[\mathrm{Ne} \mathrm{v}]$} & ${ }^{3} \mathrm{P}_{1}-{ }^{3} \mathrm{P}_{0}$ & $4.6 \pm 0.3$ & $4.7 \pm 0.6$ \\
25.8903 & {$[\mathrm{O} \mathrm{IV}]$} & ${ }^{2} \mathrm{P}_{3 / 2}-{ }^{2} \mathrm{P}_{1 / 2}$ & $7.0 \pm 0.2$ & $6.4 \pm 0.1$ \\
\hline \hline
\end{tabular}

${ }^{a}$ Blended.

The emerging picture of the the dust distribution in HM Sge is thus that of a partial Mira dust shell that is not too different from those of single Miras but in addition, there is a thin dust-rich layer at the interface between the Mira dust shell and the dust-free region carved out by the hot component. It is this geometrically thin shell that makes a symbiotic a D-type. The "M" shell only marginally affects the stellar spectrum at JHK. The proposed model not only fits the ISO spectrum, but also easily accomodates the facts that (a) HM Sge suffered a dust obscuration event (see discussion), (b) the red giant is no longer very deeply embedded in a dust shell and (c) the radio data (Kwok et al. 1984).

\subsection{Caveats}

While reasonable fits to the data have been obtained, we should note some caveats:

(a) DUSTY implicitly assumes that the situation being modelled is an equilibrium state, but it is not clear that this applies to the case of HM Sge because of the relatively rapid changes that are occurring in the Mira component. The thermal inertia of the grains ensures that they will reach equilibrium with the radiation field on a time-scale that is negligible by comparison with the pulsation period of the Mira; however there may be bulk motions in the dust shell as a result of the Mira pulsations and wind interactions which DUSTY does not take into account.

(b) Richards et al. (1999) determine the binary separation to be $\sim 25$ AU $\sim 3.8 \times 10^{14} \mathrm{~cm}$. With a dust condensation tempertature of $1500 \mathrm{~K}$ the inner boundary of the Shell $\mathrm{M}$ is $\sim 4$ stellar radii from the stellar photosphere so that the outer boundary is $\sim 4000$ stellar radii $\sim 5 \times 10^{16} \mathrm{~cm}$. It is therefore an ad hoc assumption that there are two separate dust shells, although the configuration deduced by Kwok et al. (1984) from radio observations shows that this approximation is a good one. Nonetheless a more meaningful treatment would have the hot component embedded in the red giant dust shell, but this is beyond the scope of current versions of DUSTY.

(c) There is only radiative heating of the dust and each star heats only its own shell.

Despite these caveats, we emphasize that the model fit presented here is by far the most sophisticated treatment of the HM Sge dust shell to date.

\section{Emission lines}

A number of emission lines are clearly detected in the SWS wavelength range, and which were seen in both observations; these are listed in Table 3 . In order to determine the fluxes the lines were fitted with Gaussians and the flux errors given in Table 3 are the formal errors from the fit. In general these seem consistent with the uncertainties to be expected on the basis of the absolute calibration accuracy of the SWS (Leach et al. 2000). Where upper limits are given they are $3 \sigma$. We conclude that there is no evidence for line flux changes between the two observations, within the quoted accuracy. At shorter (optical/near IR) wavelengths the line widths are typically $\sim 100-200 \mathrm{~km} \mathrm{~s}^{-1}$ (Solf 1984; Mueller \& Nussbaumer 1985). At the resolution of SWS-01 speed 1 observations the lines are not resolved.

We note that $\mathrm{HI} \mathrm{Br}-\alpha \lambda 4.0522$ is detected in both observations but we do not see $\mathrm{H}$ I $\mathrm{Hu}-\alpha \lambda 12.3719$ to a limit of $5 \times 10^{-15} \mathrm{~W} \mathrm{~m}^{-2}$. However this is not surprising because, at an electron temperature of $10^{4} \mathrm{~K}$, and assuming that both transitions are optically thin, the flux ratio is in the range $\sim 6.5-10$ for electron densities in the range $10^{4}-10^{10} \mathrm{~cm}^{-3}$ (Storey \& Hummer 1995). The predicted Hu- $\alpha$ flux is therefore $\sim 10^{-15} \mathrm{~W} \mathrm{~m}^{-2}$.

The spectrum is dominated by high-ionization forbidden lines, particularly of $\mathrm{Ne}$ (see Table 3 ) and it is evident that high ionization neon lines (such as [Ne v] and [Ne VI]) are much stronger than those of low ionization (such as $[\mathrm{Ne} I \mathrm{I}])$. We defer an analysis of the emission lines to a later paper.

\section{Discussion}

We have presented ISO SWS/LWS observations of the symbiotic star HM Sge, obtained at a time when the dust shell formed in 1985 was beginning to disperse. We have made the first attempt at a detailed fit to the dust emission, and find that the deduced circumstellar dust shells are broadly consistent with the known properties of HM Sge.

We favour a model of two dust components: An extended but optically thin shell typical for a red giant is combined with a thin dust rich layer. This combination of shells may well be responsible for the defining criterion of D-type symbiotic systems i.e. noticeable dust emission 
at JHK. The 2-component model can physically be justified with the following scenario: A strong UV light source placed in the expanding dust shell of a red giant will firstly destroy the dust grains and generate an ionised nebulosity. Radiation pressure from the companion will secondly slow the red giant wind and generate a compression wave in the transition zone. In this compression wave the conditions for dust formation are very favourable and we can identify our thin dust rich layer with this structure. The compression can still be increased if the hot companion has its own wind and a layer of shocked material is generated in a wind-wind collision zone. Such a scenario is supported by X-ray observations (Mürset et al. 1997) which demonstrate a hot plasma of low luminosity is present. The question of how dust can be formed in such an environment is an interesting one that has already been addressed in the context WR+O binaries (e.g. Marchenko et al. 1999; Folini \& Walder 2000a) that occasionally suffer prolific and episodic dust formation events. Simulations of wind collision zones show that, inside the collision zone, an unstable, radiatively cooled high density layer forms (Folini \& Walder 2000b). In this layer large density and temperature gradients occur such that pockets of dust formation seem possible. In this context it is interesting to note that our dust models are consistent with pure radiation heating of the dust and do not need additional collsional heating.

There still remain discrepancies between our models and the observations, which presumably can be resolved by more refined modelling and the inclusion of more appropriate stellar atmospheres, dust shell geometries and perhaps silicate optical constants.

We also see, as at shorter wavelengths, a strong emission line spectrum, dominated by high ionization states of $\mathrm{Ne}, \mathrm{Si}, \mathrm{Fe}$ and $\mathrm{Mg}$. A complete understanding of the ISO spectrum of HM Sge must include rigorous and selfconsistent modelling of both dust shell and emission line region.

Acknowledgements. We thank our colleague Dr. Thomas Dumm for fruitful comments on the manuscript. S. P. S. E. was supported by the UK Particle Physics and Astronomy Research Council. Based on observations with ISO, an ESA project with instruments funded by ESA Member States (especially the PI countries: France, Germany, the Netherlands and the UK) with the participation of ISAS and NASA.

\section{References}

Anandarao, B. G., Taylor, A. R., \& Pottasch, S. R. 1988, A\&A, 203, 361

Burgdorf, M., et al. 1997, Proc. of the First ISO workshop on analytical spectroscopy, ESA SP-419, 51

Clegg, P. E., et al. 1996, A\&A, 315, L38

Corradi, R. L. M. 1995, MNRAS, 276, 521

de Graauw, Th. et al. 1996, A\&A, 315, L49

Eyres, S. P. S., Evans, A., Salama, A., et al. 1998, in Proc. of ISO's view on stellar evolution, ed. L. B. F. M. Waters, C. Waelkens, K. A. van der Hucht, \& P. A. Zaal (Kluwer Academic Publishers), 361
Eyres, S. P. S., Bode, M. F., Taylor, A. R., Crocker, M. M., \& Davis, R. J. 2001, ApJ, 551, 512

Feast, M. W., Glass, I. S., Whitelock, P. A., \& Catchpole, R. M. 1989, MNRAS, 241, 375

Feast, M. W. 1996, MNRAS, 278, 11

Folini, D., \& Walder, R. 2000a, in Thermal and Ionization Aspects of Flows from Hot Stars: Observations and Theory, ed. J. G. L. M. Lamers, \& A. Sapar, ASP Conf. Ser., 204, 267

Folini, D., \& Walder, R. 2000b, A\&A, submitted

Fluks, M. A., Plez, B., The P. S., et al. 1994, A\&AS, 105, 311

Habing, H. J. 1996, A\&A Rev., 7, 97

Ivezić, Z., \& Elitzur, M. 1997, MNRAS, 287, 799 http://www.pa.uky.edu/ ${ }^{\text {moshe/dusty/ }}$

Kamath, U. S., \& Ashok, N. M. 1999, A\&AS, 135, 199

Kessler, M., et al. 1996, A\&A, 315, L27

Kwok, S., Bignell, R. C., \& Purton, C. R. 1984, ApJ, 279, 188

Leach, K., et al. 2000, ISO Handbook, vol. VI: SWS

Lejeune, Th., Cuisinier, F., \& Buser, R. 1997, A\&AS, 125, 229

Lobel, A., Bagnulo, S., Doyle, J. G., \& Power, C. 2000, MNRAS, 317, 391

Lutz, J. H., et al. 1989, PASP, 101, 966

Marchenko, S. V., Moffat, A. F. J., \& Grosdidier, Y. 1999, ApJ, 522,433

Mathis, J. S., Rumpl, W., \& Nordsieck, K. H. 1977, ApJ, 217, 425

Mikołajewska, J. 1997, in Physical Processes in Symbiotic Binaries and Related Systems, ed. J. Mikołajewska (Copernicus Found. for Polish Astronomy, Warsaw), 3

Mueller, B. E. A., \& Nussbaumer, H. 1985, A\&A, 145, 144

Munari, U., \& Whitelock, P. A. 1989, MNRAS, 237, 45

Munari, U., \& Renzini, A. 1992, ApJ, 397, L87

Mürset, U., \& Schmid, H. M. 1999, A\&AS, 137, 473

Mürset, U., Wolff, B., \& Jordan, S. 1997, A\&A, 319, 201

Mürset, U., Nussbaumer, H., Schmid, H. M., \& Vogel, M. 1991, A\&A, 248, 458

Ossenkopf, V., Henning, Th., \& Mathis, J. S. 1992, A\&A, 261, 567

Richards, A. M. S., Bode, M. F., Eyres, S. P. S., et al. 1999, MNRAS, 305, 380

Salama, A., et al. 1997, Proc. of the First ISO workshop on analytical spectroscopy, ESA SP-419, 17

Schild, H., Boyle, S., \& Schmid, H. M. 1992, MNRAS, 258, 95

Schild, H., \& Vogel, M. 1998, In Proc. of ISO's view on stellar evolution, ed. L. B. F. M. Waters, C. Waelkens, K. A. van der Hucht, \& P. A. Zaal (Kluwer Academic Publishers), 367

Schild, H., Dumm, T., Folini, D., Hussbaumer, H., \& Schmutz, W. 1999, in Proc. of The Universe as seen by ISO, ed. P. Cox, \& M.F. Kessler, ESA SP-427, 397

Schmid, H. M., Corradi, R., Krautter, J., \& Schild, H. 2000, A\&A, 355, 261

Solf, J. 1984, A\&A, 139, 296

Storey, P. J., \& Hummer, D. G. 1995, MNRAS, 272, 41

VSNET 1999, http://www.kusastro.kyoto-u.ac.jp/vsnet/ LCs/ index/sgehm.html

Whitelock, P. A. 1987, PASP, 99, 573

Yudin, B., Munari, U., Taranova, O., \& Dalmeri, I. 1994, A\&AS, 105, 169 